\title{
Editorial: advanced methods for public transport system management
}

\author{
Hong K. Lo • William H.K. Lam • S.C. Wong • \\ Janny M.Y. Leung
}

Published online: 22 July 2010

(C) The Author(s) 2010. This article is published with open access at Springerlink.com

As the world is pressed with energy crises and environmental concerns, to address the mobility needs in urban areas, there is a resurgence of interest in encouraging more wide-spread use of public transport in lieu of private cars. The benefits of public transport are many: increased mobility for all, reduced car dependence and consequential needs for highway expansions. The success of this proposition, however, hinges largely on whether public transport offers attractive alternatives to private car usage. It is ideal if public transport services are financially sustainable, with affordable fares, and expedient quality. Despite many decades of intense effort, methods for public transport planning and operations are still in their infancy of development and refinement, as evidenced by the fast pace of developments in both theory and practice.

H.K. Lo (ه)

Department of Civil and Environmental Engineering, The Hong Kong University of Science and Technology, Clear Water Bay, Kowloon, Hong Kong

e-mail: cehklo@ust.hk

W.H.K. Lam

Department of Civil and Structural Engineering, The Hong Kong Polytechnic University, Hung Hom, Kowloon, Hong Kong

e-mail: cehklam@inet.polyu.edu.hk

S.C. Wong

Department of Civil Engineering, The University of Hong Kong, Pokfulam Road, Hong Kong,

Hong Kong

e-mail: hhecwsc@hkucc.hku.hk

J.M.Y. Leung

Department of Systems Engineering and Engineering Management, The Chinese University

of Hong Kong, Shatin, N.T., Hong Kong

e-mail: jleung@se.cuhk.edu.hk 
This is the first of several special issues based on papers selected from the 11th International Conference on Advanced Systems for Public Transport (CASPT) held in Hong Kong on 20-22 July 2009. CASPT serves as a forum for the international community of researchers and practitioners on all aspects of public transport planning and operations. Previous CASPT conferences were held at: Leeds, 2006, San Diego, 2004, Berlin, 2000, Boston, 1997, Lisbon, 1993, Montreal, 1990, Hamburg, 1987, Montreal, 1983, Leeds, 1980, Chicago, 1975. To reflect the expanded scope of this conference series, it has taken on the new title "Conference on Advanced Systems for Public Transport" from its previous title "Computer-Aided Scheduling of Public Transport". CASPT covers significant contributions to the theory and application of systems and methodologies for advancing public transport planning and operations.

This special issue covers innovations in public transport management, ranging from studying the feasibility of demand responsive services based on vehicle automation, better crew scheduling that incorporates the crew's annual vacation constraints, robust routing of rapid transit rolling stock, transit services planning under network uncertainty, to the use of road pricing as a policy to encourage modal shifts to public transport. Recently, the thrust of public transport development has emerged strongly in Asia, wherein the public transport system, often operated by the private sector, plays a pivotal role in serving the mobility needs of many major cities. Hence, new aspects on public transport management and operations arise. For example, the planning of public transport must take into account the long-term financial viability of these services, as well as the forms of public private partnership that would be most suited for their implementation. The other aspect is the question of how the roadway environment, with a mixture of heterogeneous types of traffic, would affect the performance of buses. Understanding these aspects would allow for the design of more serviceable systems, financially and operationally. These papers well illustrate the range of endeavors to extend the state-of-the-art and practice. Seven papers are collected in this first special issue, as summarized below.

The paper by Alessandrini, Filippi, Stam, and Tripodi develops a pre-design method to study a new generation of urban transport systems that can supply carlike quality of service with public transport-like impacts. The authors then apply the approach to simulate nearly 3000 scenarios and perform statistical regressions on the results. Empirical validations, by comparing the pre-design regressions with other system data, showed that the method gives accurate results.

The paper by Dewess studies the problem of crew scheduling while considering socially acceptable annual holiday planning. The new model takes into account legal, company and driver issues, including capacity constraints concerning different qualifications, holiday entitlements and connections between drivers. The problem is then solved by a two-stage heuristic algorithm. Computational results show that the algorithm is efficient for solving problems up to 10,000 drivers.

The paper by Cadarso and Marin develops a robust model to manage the rolling stock resources for situations where the service frequencies are high and travel distances are relatively short. The model minimizes the delay propagation in each sequence of rolling stock assignment as well as crew requirements at depot stations. Computational experiments are developed for the Madrid suburban rail network. The results show that robust and efficient solutions can be obtained, as compared with the current solutions. 
The paper by Zhang, Lam, Sumalee, Lo, and Tong proposes a new multi-class user reliability-based dynamic transit assignment model that incorporates both network demand and supply uncertainties. Furthermore, passengers are modeled to exhibit heterogeneous risk-taking attitudes towards random travel costs, hence influencing their departure time, mode and route choices. The proposed model is formulated as a fixed-point problem, and solved by a heuristic MSA-type algorithm. The numerical result shows that travelers' risk-taking attitude would greatly impact passengers' travel mode and departure time choices.

The paper by Tsekeris and Voß surveys and studies the interrelationships between public transport and road pricing, covering both theoretical and empirical aspects. After reviewing the main decision parameters, the paper develops methodologies to study policies that aim at improving urban public transport ridership and reducing private vehicle use. The experiments focus on the hypothetical implementation of a national distance-based road charging policy package in Greece. The simulation results indicate that a suitably designed policy package can significantly enhance modal split in favor of public transport without significantly increasing the level of road user charges.

The paper by Tang and Lo studies different forms of public private partnerships for the provision of mass railway transit services. The study develops influence diagrams to assess and portray how the roles of different parties in developing mass railway transit may influence the accomplishment of financial, transportation and construction objectives of a project. The paper then applies the approach to rail transit systems in Hong Kong and elsewhere to identify advantages and potential pitfalls of different public and private partnerships in meeting the objectives of the projects.

The paper by Arasan and Vedagiri studies and quantifies possible impacts of providing an exclusive bus lane under heterogeneous traffic conditions. Through a simulation model developed for heterogeneous traffic, the paper studies improvements in the level of service due to the exclusive bus lane, as well as impacts on other types of motor vehicles due to the consequent reduction in road space. The study then recommends a certain maximum permissible volume to capacity ratio that would ensure acceptable levels of service for the traffic stream as a whole comprising different types of motor vehicles.

The papers published in this special issue are by no means an exhaustive collection of emerging efforts for improving the management of public transport systems. However, we trust that they do provide timely reviews of recent methodological advances. It is our hope that this special issue will inspire and stimulate new research initiatives and efforts in this still evolving field.

Open Access This article is distributed under the terms of the Creative Commons Attribution Noncommercial License which permits any noncommercial use, distribution, and reproduction in any medium, provided the original author(s) and source are credited. 